\title{
X-ray Crystal Structure of Aristolochene Synthase from Aspergillus terreus and the Evolution of Templates for the Cyclization of Farnesyl Diphosphatet,‡
}

\author{
Ekaterina Y. Shishova§, Luigi Di Costanzo§, David E. Canell, and David W. Christianson ${ }^{\star}, \S$ \\ Roy and Diana Vagelos Laboratories, Department of Chemistry, University of Pennsylvania, \\ Philadelphia, Pennsylvania 19104-6323, and Department of Chemistry, Brown University, \\ Providence, Rhode Island 02912
}

\begin{abstract}
Aristolochene synthase from Aspergillus terreus catalyzes the cyclization of the universal sesquiterpene precursor, farnesyl diphosphate, to form the bicyclic hydrocarbon aristolochene. The $2.2 \AA$ resolution $\mathrm{X}$-ray crystal structure of aristolochene synthase reveals a tetrameric quaternary structure in which each subunit adopts the $\alpha$-helical class I terpene synthase fold with the active site in the "open", solvent-exposed conformation. Intriguingly, the $2.15 \AA$ resolution crystal structure of the complex with $\mathrm{Mg}^{2+}{ }_{3}$-pyrophosphate reveals ligand binding only to tetramer subunit $\mathrm{D}$, which is stabilized in the "closed" conformation required for catalysis. Tetramer assembly may hinder conformational changes required for the transition from the inactive open conformation to the active closed conformation, thereby accounting for the attenuation of catalytic activity with increasing enzyme concentration. In both conformations, but especially so in the closed conformation, the active site contour is highly complementary in shape to that of aristolochene, and a catalytic function is proposed for the pyrophosphate anion based on its orientation with regard to the presumed binding mode of aristolochene. A similar active site contour is conserved in aristolochene synthase from $P$. roqueforti despite the substantial divergent evolution of these two enzymes, while strikingly different active site contours are found in the sesquiterpene cyclases 5-epi aristolochene synthase and trichodiene synthase. Thus, the terpenoid cyclase active site plays a critical role as a template to bind the flexible polyisoprenoid substrate in the proper conformation for catalysis. Across the greater family of terpenoid cyclases, this template is highly evolvable within a conserved $\alpha$-helical fold for the synthesis of terpene natural products of diverse structure and stereochemistry.
\end{abstract}

The terpenoid family consists of tens of thousands of structurally and stereochemically complex natural products that ultimately derive from a mere handful of linear isoprenoid precursors. For example, cyclic sesquiterpenes comprise a broad family of $\mathrm{C}_{15}$-isoprenoids that serve myriad biological functions in plants, bacteria, and fungi $(1,2)$, yet each sesquiterpene derives from the universal sesquiterpene precursor, farnesyl diphosphate, through a reaction catalyzed by a sesquiterpene cyclase (2-9). In general, a terpene cyclase governs a specific cyclization cascade with exquisite precision to generate a single, unique product. However,

\footnotetext{
${ }^{\dagger}$ This work was supported by National Institutes of Health Grants GM 56838 (D.W.C.) and GM 30301 (D.E.C.)

\$Atomic coordinates of aristolochene synthase from A. terreus and its complex with $\mathrm{Mg}^{2+}{ }_{3}$-pyrophosphate have been deposited in the Protein Data Bank with accession codes 2E4O and 2OA6, respectively.

*To whom correspondence should be addressed at the Roy and Diana Vagelos Laboratories, Department of Chemistry, University of Pennsylvania, 231 South $34^{\text {th }}$ St., Philadelphia, Pennsylvania 19104-6323. Phone: 215-898-5714. Fax: 215-573-2201. E-mail:

chris@sas.upenn.edu.

\$University of Pennsylvania.

\|Brown University.
} 
some terpene cyclases are somewhat promiscuous and generate complex mixtures of cyclization products (10).

To date, crystal structures of four sesquiterpene cyclases have been determined: 5-epiaristolochene synthase from Nicotiana tabacum (11), pentalenene synthase from Streptomyces UC5319 (12), aristolochene synthase from Penicillium roqueforti (13), and trichodiene synthase from Fusarium sporotrichioides (14). Despite the general lack of significant amino acid sequence identity among these cyclases, their three-dimensional structures share a common $\alpha$-helical fold $(5,6,9)$, as well as two metal binding motifs conserved among monoterpene, sesquiterpene, and diterpene cyclases: the so-called "aspartate-rich" motif DDXXD/E of helix D (15), and the "NSE/DTE" motif $(\mathrm{L}, \mathrm{V})(\mathrm{V}, \mathrm{L}, \mathrm{A})(\mathbf{N}, \mathbf{D}) \mathrm{D}(\mathrm{L}, \mathrm{I}, \mathrm{V}) \mathrm{X}$ $(\mathbf{S}, \mathbf{T}) \mathrm{XXXE}$ of helix $\mathrm{H}$, in which boldface residues are usually magnesium ligands $(14,16)$. A sesquiterpene cyclase initiates catalysis by the metal ion-dependent ionization of farnesyl diphosphate to generate inorganic pyrophosphate and a highly reactive allylic carbocation.

Aristolochene synthase catalyzes the cyclization of farnesyl diphosphate to form the bicyclic sesquiterpene (+)-aristolochene. The 36-kDa enzyme from Aspergillus terreus shares $61 \%$ amino acid sequence identity with the $38-\mathrm{kDa}$ aristolochene synthase from $P$. roqueforti (13). In $A$. terreus and $P$. roqueforti, aristolochene formation is the first committed step in the biosynthesis of a group of eremophilene toxins and bioregulators such as PR toxin, sporogenAO 1, phomenone, and bipolaroxin (16). Extensive mechanistic studies on both $P$.

roqueforti and $A$. terreus enzymes using stereospecifically-labelled farnesyl diphosphate, the anomalous substrate 6,7-dihydrofarnesyl diphosphate, a mechanism-based inhibitor, and sitespecific mutants (17-21), support a common cyclization mechanism (Figure 1) in which farnesyl diphosphate ionization is accompanied by electrophilic attack on the $\mathrm{C} 10-\mathrm{C} 11 \pi$ bond, with inversion of configuration at $\mathrm{C} 1$, resulting in the formation of the neutral hydrocarbon intermediate 2, $(S)$-(-)-germacrene A. Protonation at $\mathrm{C} 6$ and electrophilic attack of the resulting $\mathrm{C} 7$ carbocation on the $\mathrm{C} 2-\mathrm{C} 3 \pi$ bond generate the eudesmane cation $\mathbf{3}$, which after methyl migration, hydride transfer, and stereospecific deprotonation yields (+)-aristolochene 4.

Here, we report the X-ray crystal structures of recombinant aristolochene synthase from Aspergillus terreus at $2.2 \AA$ resolution and its complex with inorganic pyrophosphate $\left(\mathrm{PP}_{\mathrm{i}}\right)^{1}$ and $3 \mathrm{Mg}^{2+}$ ions at $2.15 \AA$ resolution. Comparison of these structures reveals tertiary structural changes required for catalysis and quaternary structural changes that may attenuate catalytic activity with increasing enzyme concentrations. Moreover, the structural comparison of aristolochene synthases from $A$. terreus and $P$. roqueforti reveals the conservation of a unique active site contour complementary in shape to their common product despite the substantial divergent evolution of these two enzymes. Finally, comparisons of these cyclases with 5-epiaristolochene synthase and trichodiene synthase reveal striking differences in active site contours, thereby illustrating the biological function of terpene cyclases as both specific and highly evolvable templates for the synthesis of terpene natural products of diverse structure and stereochemistry.

\section{Materials and Methods}

Recombinant aristolochene synthase from A. terreus was expressed at high levels in Escherichia coli BL21(DE3)pLysS (5-10\% of total protein) and purified as previously described (16) with minor modifications. Briefly, E. coli BL21(DE3)pLysS carrying pET11rASA was inoculated into Luria-Bertani media containing carbenicillin and grown overnight at $37^{\circ} \mathrm{C}$. A total of $500 \mathrm{~mL}$ of LB-carbenicillin media was inoculated with $5 \mathrm{~mL}$ of the overnight

\footnotetext{
${ }^{1}$ Abbreviations: inorganic pyrophosphate, $\mathrm{PP}_{\mathrm{i}} ; \beta$-mercaptoethanol, BME; 2-morpholinoethanesulfonic acid, MES; noncrystallographic symmetry, NCS.
} 
seed culture and $E$. coli was grown at $30^{\circ} \mathrm{C}$ until $\mathrm{OD}_{600 \mathrm{~nm}}=0.7$. Following induction with $0.5 \mathrm{mM}$ isopropyl- $\beta$-D-thiogalactopyranoside for $4 \mathrm{~h}$, cells were harvested, resuspended in 50 $\mathrm{mL}$ of DE52 equilibration buffer (20 mM 2-morpholinoethanesulfonic acid (MES) (pH 6.5), $5 \mathrm{mM}$ ethylenediaminetetraacetic acid, $5 \mathrm{mM} \beta$-mercaptoethanol (BME), $10 \%$ glycerol), and sonicated for $10 \mathrm{~min}$ using a 50\% duty cycle and power range of 30\%. After three cycles of sonication, cell lysate was clarified by centrifugation at $17,000 \mathrm{~g}$ at $4{ }^{\circ} \mathrm{C}$ for $30 \mathrm{~min}$. The clarified lysate was loaded on DE52 anion exchange resin $(80 \mathrm{~mL})$ and a linear gradient of $0-500 \mathrm{mM}$ $\mathrm{NaCl}$ in cell lysis buffer was applied to elute the enzyme. The most active fractions were pooled, mixed with an equal volume of $3 \mathrm{M}$ ammonium sulfate in DE52 buffer, and applied to a 15 $\mathrm{mL}$ methyl HIC column. The enzyme was eluted with a linear gradient of $1.5-0 \mathrm{M}$ ammonium sulfate in the same buffer. The most active fractions were desalted on a Sephadex G-25 column and further purified with anion exchange chromatography, using a $10 \mathrm{~mL}$ High Q column and a linear gradient of $0-300 \mathrm{mM} \mathrm{NaCl}$ in DE52 buffer. The resulting protein preparation was $85-90 \%$ purity based on SDS-PAGE.

The native enzyme was crystallized by the hanging drop vapor diffusion method at $4^{\circ} \mathrm{C}$. Typically, a $5 \mu \mathrm{L}$ drop of protein solution [ $5 \mathrm{mg} / \mathrm{mL}$ aristolochene synthase, $25 \mathrm{mM}$ MES ( $\mathrm{pH}$ 6.5), $2 \mathrm{mM} \mathrm{MgCl} 2,0.15 \mathrm{M} \mathrm{NaCl}, 4 \mathrm{mM} \mathrm{BME}$ ] was added to $5 \mu \mathrm{L}$ of precipitant solution [100 $\mathrm{mM}$ Tris $\mathrm{HCl}(\mathrm{pH} 8.4), 18 \%$ PEG 6000, $0.5 \mathrm{M} \mathrm{NaCl}]$ and equilibrated against a $1 \mathrm{~mL}$ well reservoir of precipitant solution. Crystals appeared within 3-4 weeks and grew to maximal dimensions of $0.15 \mathrm{~mm} \times 0.09 \mathrm{~mm} \times 0.02 \mathrm{~mm}$. X-ray diffraction data were collected from these crystals at beamline X29A at the Brookhaven National Synchrotron Light Source and processed to $2.2 \AA$ resolution using the HKL suite of programs (22). Crystals belong to space group $P 21_{1}$ with unit cell parameters $a=61.2 \AA, b=147.2 \AA, c=83.7 \AA$, $\beta=97.9^{\circ}$. With 4 molecules in the asymmetric unit, the Matthew's coefficient $\mathrm{V}_{\mathrm{m}}=2.6 \AA^{3} / \mathrm{Da}$, corresponding to a solvent content of $52 \%$.

Initial phases for the electron density map of $A$. terreus aristolochene synthase were determined by molecular replacement using the structure of aristolochene synthase from $P$. roqueforti as a search probe for rotation and translation functions as implemented in the program Phaser 1.3 (23). After placement of four monomers in the asymmetric unit, rigid-body refinement yielded $\mathrm{R} / \mathrm{R}_{\text {free }}=0.460 / 0.457$. Iterative rounds of refinement and model adjustment were performed using CNS (24) and O (25), respectively. Iterative cycles of refinement utilized torsion angle dynamics with a starting temperature of $5,000 \mathrm{~K}$ in the initial stages of refinement with tight noncrystallographic symmetry (NCS) restraints relating the four monomers. With some differences among subunits being apparent, the NCS restraints were loosened as and ultimately dropped for the last cycles refinement progressed as guided by $\mathrm{R}_{\text {free }}$ refinement. The $\mathrm{N}$-termini (M1-S12), C-termini (V318-D320), and loop segments S231-G239 were disordered in all four monomers; thus, these residues are excluded from the final model. Continuous electron density peaks adjacent to five cysteine side chains were modeled as disulfide-linked BME molecules. Strong electron density peaks adjacent to R314 side chains in monomers B and C were interpreted as chloride ions. A large and continuous electron density peak in the active site cavity of monomer D was interpreted as a molecule of the buffer MES with its sulfonate group salt linked to the R314 side chain.

Individual thermal B factors were refined and refinement ultimately converged to a final crystallographic $R$-factor of $0.222\left(R_{\text {free }}=0.272\right)$ with excellent stereochemistry. Data collection and refinement statistics are summarized in Table 1 . The buried surface area between monomers was calculated using the method of Lee and Richards with a $1.4 \AA$ probe (26), as implemented in CNS (24).

The structure of A. terreus aristolochene synthase complexed with $\mathrm{Mg}^{2+}{ }_{3}-\mathrm{PP}_{\mathrm{i}}$ was determined by soaking crystals of the native enzyme for 20 hours in a buffer solution containing $100 \mathrm{mM}$ 
Tris $\mathrm{HCl}$ (pH 8.4), 18\% PEG 6000, $0.5 \mathrm{M} \mathrm{NaCl}, 10 \mathrm{mM}$ sodium pyrophosphate, $5 \mathrm{mM}$ $\mathrm{MgCl}_{2}$, and $5 \mathrm{mM}$ benzyl tetraethyl ammonium chloride. X-ray diffraction data from these crystals were collected at the Cornell High Energy Synchrotron Source, beamline F-1, and processed to $2.15 \AA$ resolution using the HKL suite of programs (22). Crystals were isomorphous with those of the native enzyme. The initial difference Fourier map revealed the binding of inorganic pyrophosphate and three $\mathrm{Mg}^{2+}$ ions to monomer D only. Significant conformational changes were evident in monomer $\mathrm{D}$ and the polypeptide chain was rebuilt accordingly. Some attenuated conformational changes, but no ligand binding, were also observed in monomer $\mathrm{C}$. Iterative rounds of refinement and model adjustment were performed using CNS (24) and O (25), respectively. The N-termini (M1-S12) and C-termini (V318D320) were disordered in all four monomers, and the loop segments S231-G239 were disordered in monomers A, B, and C. The loop segment N48-A51 was disordered in monomer C. The S231-G239 loop segment was ordered in monomer D to accommodate the binding of $\mathrm{Mg}^{2+}{ }_{3}-\mathrm{PP}_{\mathrm{i}}$. Continuous electron density peaks adjacent to four cysteine side chains were modeled as disulfide-linked BME molecules. Individual thermal B factors were refined and a bulk-solvent correction was applied. Refinement ultimately converged to a final crystallographic $R$-factor of $0.229\left(R_{\text {free }}=0.273\right)$ with excellent stereochemistry. Data collection and refinement statistics are summarized in Table 1. Molecular surfaces in the figures are calculated with Voidoo (27).

\section{Results}

Aristolochene synthase from A. terreus adopts the $\alpha$-helical class I terpene synthase fold (Figure 2a) first observed for avian farnesyl diphosphate synthase (28). There are total of 13 $\alpha$-helices, 6 of which surround the $20 \AA$ A-deep active site cleft. Most of the helices are joined by short loops, about 4-5 residues in length. Two 10-residue loops connect helices D1 and E and helices F and G1, and a partly-disordered 16-residue loop connects helices $\mathrm{H}$ and $\alpha-1$. The aspartate-rich DDLLE motif beginning with D90 is located on the C-terminal end of helix D on the upper wall of the active site cleft, and the NSE/DTE motif starting with V217 is located on helix $\mathrm{H}$ on the opposite wall of the active site cleft. No metal ions are observed bound to these motifs in the structure of the unliganded enzyme.

Aristolochene synthase from A. terreus is reported to be a monomer in solution based on the results of gel filtration chromatography (16). However, native gel analysis indicates dimeric quaternary structure (Figure 3), and the crystal structure reveals the assembly of two dimers in the asymmetric unit to form a tetramer with 222 point group symmetry (Figures $2 b$ and $c$ ). This is the first example of tetrameric quaternary structure in the greater family of terpenoid cyclases. The total surface areas of individual monomers and the buried surface areas between monomers are presented in Table 2. The $\mathrm{AD}$ and $\mathrm{BC}$ monomer pairs are each related by noncrystallographic symmetry and form the largest interface with $1634 \AA^{2}$ average buried surface area. This interface is enriched with numerous hydrophobic side chains, as well as polar residues (E251, E259, D253, and R273) that form direct and solvent mediated intersubunit hydrogen bonds. The AC and BD monomer pairs each form a significant, somewhat polar interface with $1009 \AA^{2}$ average buried surface area. Finally, the $A B$ and CD monomer pairs bury an average of $720 \AA^{2}$ of polar and nonpolar surface area. In total, each monomer buries approximately $23 \%$ of its total surface area upon tetramer formation, which corresponds to the average buried surface area of $23 \%$ per subunit said to characterize homotetramer assembly (29). Therefore, we conclude that A. terreus aristolochene synthase likely functions as the AD or BC dimer in solution, but it is capable of oligomerization at higher enzyme concentrations to form a tetramer.

Such oligomerization may be responsible for the previously documented attenuation of enzyme activity at enzyme concentrations greater than $27 \mathrm{nM}(21)$, e.g., by hindering conformational 
changes required for catalysis. In light of this observation, it is intriguing that although the structure of $A$. terreus aristolochene synthase complexed with $\mathrm{Mg}^{2+}{ }_{3}-\mathrm{PP}_{\mathrm{i}}$ is the first structure of a ligand complex with an aristolochene synthase from any species, the $\mathrm{Mg}^{2+}{ }_{3}-\mathrm{PP}_{\mathrm{i}}$ cluster binds only to subunit D of the tetramer (Figure 4a). Furthermore, X-ray crystallographic analysis of enzyme crystals soaked in solutions containing farnesyl diphosphate reveals the binding of intact substrate molecules in the active sites of monomers A-C and just $\mathrm{PP}_{\mathrm{i}}$ in the active site of monomer $\mathrm{D}$ (study in progress). Accordingly, substrate-induced conformational changes required for active site closure and catalysis may indeed be hindered in monomers A$\mathrm{C}$ of the tetramer.

In the aristolochene synthase- $\mathrm{Mg}^{2+}{ }_{3}-\mathrm{PP}_{\mathrm{i}}$ complex, the carboxylate of $\mathrm{D} 90$ (the first residue of the aspartate-rich motif on helix D) coordinates to $\mathrm{Mg}^{2+}{ }_{\mathrm{A}}$ and $\mathrm{Mg}^{2+} \mathrm{C}$ with syn, syn-bidentate geometry. No other residues in the aspartate-rich motif participate in metal binding: D91 makes a salt bridge interaction with R314, and D94 accepts hydrogen bonds from water molecules $\# 1$ (coordinated to $\mathrm{Mg}^{2+} \mathrm{A}$ ) and \#66. In addition to D90, five other ligands coordinate to $\mathrm{Mg}^{2+} \mathrm{A}$, including the $\mathrm{PP}_{\mathrm{i}}$ anion and four water molecules (\#4, \#70, \#71, and \#72). The $\mathrm{Mg}^{2+} \mathrm{C}$ ion is coordinated by $\mathrm{D} 90, \mathrm{PP}_{\mathrm{i}}$, and water molecules \#1, \#3, and \#70. Adjacent to the $\mathrm{Mg}^{2+}{ }_{3}-\mathrm{PP}_{\mathrm{i}}$ cluster is a glycerol molecule that makes hydrogen bond interactions with metalcoordinated water molecules \#4 and \#70. In the NSE/DTE motif, N219, S223, and E227 coordinate to $\mathrm{Mg}^{2+}{ }_{\mathrm{B}}$ in a fashion identical to that observed in other terpenoid cyclases such as 5 -epi-aristolochene synthase (11), trichodiene synthase (14), and bornyl diphosphate synthase (30).

In addition to multiple metal coordination interactions, the $\mathrm{PP}_{\mathrm{i}}$ anion accepts hydrogen bonds from basic residues R175, K226, R314, and also Y315 (Figure 4b). These residues are analogous to R182, K232, R304, and Y305 of trichodiene synthase and make an identical constellation of interactions with $\mathrm{PP}_{\mathrm{i}}$ (Figure 4c) (14). It is remarkable that despite the massive evolutionary drift separating these two different fungal cyclases related by only $15 \%$ amino acid sequence identity, the molecular recognition of $\mathrm{Mg}^{2+}{ }_{3}-\mathrm{PP}_{\mathrm{i}}$ and the substrate diphosphate group is perfectly conserved.

It is interesting to note the interaction between D91 (the second aspartate residue in the aspartate-rich motif) and R314, which also donates a hydrogen bond to $\mathrm{PP}_{\mathrm{i}}$. In the active sites of other terpenoid cyclases, the second aspartate in the aspartate-rich motif DDXXD/E typically interacts with an arginine residue located on a neighboring helix. For example, D352 in the aspartate-rich motif of bornyl diphosphate synthase accepts a hydrogen bond from R314, which in turn donates a hydrogen bond to the $\mathrm{PP}_{\mathrm{i}}$ anion in the $\mathrm{Mg}^{2+}{ }_{3}-\mathrm{PP}_{\mathrm{i}}$ complex (30). In trichodiene synthase, D101 of the aspartate-rich motif accepts a hydrogen bond from R304, which in turn donates a hydrogen bond to the $\mathrm{PP}_{\mathrm{i}}$ anion in the $\mathrm{Mg}^{2+}{ }_{3}-\mathrm{PP}_{\mathrm{i}}$ complex (Figure 4c) (14). In addition to protein-metal ion-diphosphate interactions, aspartate-arginine-diphosphate interactions in the greater family of terpene cyclases provide the substrate with a direct connection to trigger and control protein structural changes required for the transition of the cyclase active site from the solvent-exposed "open" conformation to the solvent-sequestered "closed" conformation (Figure 4d).

The binding of $\mathrm{Mg}^{2+}{ }_{3}-\mathrm{PP}_{\mathrm{i}}$ to monomer $\mathrm{D}$ of $A$. terreus aristolochene synthase triggers significant conformational changes, such that the r.m.s. deviation of $300 \mathrm{C} \alpha$ atoms between the unliganded and liganded structures is $1.8 \AA$. The most significant changes are observed in helices $\mathrm{G} 1$ and $\mathrm{H}$, in the $\mathrm{N}$-terminus, and in the F-G1 loop (Figure 4d). The N-terminal portion of the $\mathrm{H}-\alpha-1$ loop is incorporated into an extension of helix $\mathrm{H}$, helices $\mathrm{C} 1, \mathrm{D} 1, \mathrm{~F}, \mathrm{G} 1$, and $\mathrm{H}$ shift inward, and the previously-disordered $\mathrm{H}-\alpha-1$ loop (S231-G239) adopts a well-defined conformation that caps the active site. Other secondary structural elements that undergo significant movement include helices D, F, and I. These conformational changes are likely to 
be triggered as well by the diphosphate group of the substrate, farnesyl diphosphate, thereby bringing ligand-binding residues closer together and simultaneously sequestering the active site from bulk solvent. These conformational changes must also trigger the ionization of the substrate diphosphate group so as to initiate the cyclization cascade summarized in Figure 1.

\section{Discussion}

It is notable that aristolochene synthases from $A$. terreus and $P$. roqueforti divergently evolved yet preserved the ability to cyclize farnesyl diphosphate to form the same hydrocarbon product with exquisite structural and stereochemical precision. Although the $P$. roqueforti aristolochene synthase also produces minor amounts of the intermediate germacrene $\mathrm{A}$ and the side-product valencene (4\% and $2 \%$, respectively), the A. terreus enzyme produces aristolochene as the single sesquiterpene product (21). Of course, the substitution of active site residues important for metal binding can significantly compromise catalysis and cyclization fidelity in both cyclases (21). Mutagenesis and structural studies of the related sesquiterpene cyclase trichodiene synthase demonstrate that slight alterations in a metal binding site, e.g., the substitution of a glutamate ligand for an aspartate ligand in the aspartate-rich motif (31, 32 ), or the substitution of polar residues that donate hydrogen bonds to the substrate diphosphate group (33-36), can alter the active site contour in the catalytically-active closed conformation of the cyclase sufficiently to compromise catalytic efficiency and/or lead to the formation of aberrant cyclization products. How, then, are the amino acid substitutions between aristolochene synthases from $A$. terreus and $P$. roqueforti (61\% sequence identity) accommodated so as to preserve the structural and stereochemical precision of aristolochene biosynthesis?

The overall fold of the aristolochene synthase monomer from A. terreus is similar to that of $P$. roqueforti (Figure 5a), and the root-mean-square (r.m.s.) deviation between the two structures is $1.0 \AA$ for $282 \mathrm{C} \alpha$ atoms. The majority of amino acid substitutions between the two enzymes are found on the protein surfaces (Figure 5b), although several are located within the interior of the protein. To some extent, the protein structures compensate for interior amino acid substitutions so as to preserve the overall fold (Figure 5a), either by adjustments of surrounding residues to accommodate changes in the volume and shape of mutated side chains, or by rearrangements of hydrogen bond networks. Importantly, most of the residues lining the active site clefts are highly conserved between the two enzymes, such that the active site contours are remarkably similar (Figures 6a and 6b). Although there is only modest amino acid sequence identity between the $\mathrm{H}-\alpha-1$ loop segments that cap the active sites in the two aristolochene synthases (A. terreus: $\mathrm{Y}^{229}$ TSKTAHSEGGI; P. roqueforti: $\mathrm{E}^{254}$

ASRTGHKEGAF), the key residues are conserved. Thus, K232 in the A. terreus enzyme, which interacts with the bound $\mathrm{PP}_{\mathrm{i}}$ moiety, corresponds to $\mathrm{R} 257$ in the $P$. roqueforti enzyme.

In contrast, the active site contours of trichodiene synthase and 5-epi aristolochene synthase, enzymes that generate structurally and/or stereochemically different products, are strikingly different from one another as well as from the contours of the two aristolochene synthases (Figures $6 \mathrm{c}$ and 6d). Comparison of all the active site contours illustrated in Figure 6 validates the template function postulated for the terpene cyclase active site, in which a unique surface contour enforces the precise conformation of farnesyl diphosphate required for cyclization to form a unique product. Furthermore, the template is quite complementary in shape to the cyclization product, as shown in Figure 7 for A. terreus aristolochene synthase. Interestingly, the cyclization template is defined by a handful of conserved residues in aristolochene synthases from A. terreus and $P$. roqueforti, whereas the corresponding residues have largely diverged in trichodiene synthase and pentalenene synthase. The highly variable evolutionary fate of active site residues defining the cyclization template is consistent with their designation as "plasticity residues" by Keasling and colleagues (37), who elegantly demonstrate that a 
single terpene cyclase active site can serve as a highly evolvable template for the synthesis of cyclic hydrocarbons with diverse structures and stereochemistries.

It could be argued that the active site surface contour of a terpenoid cyclase is different in the open, unliganded state compared with the closed, ligand-bound state. However, despite the obvious changes in the contour of the upper active site triggered by its closure, the salient features of the lower active site surface contour appear to be preserved in both the open and closed conformations of A. terreus aristolochene synthase (Figure 7). Furthermore, the cyclization product is unambiguously modeled into the active site surface contour of the closed conformation (Figure 7). Since the unique characteristics of the template are largely conserved in closed and open conformations, it is possible that the cyclization product of a terpenoid cyclase might ultimately be predicted or engineered based solely on analysis of the three dimensional contour of its active site in either conformation.

Given the similar active site contours of aristolochene synthases from A. terreus and $P$. roqueforti, the structural analysis and mechanistic implications discussed by Caruthers and colleagues (13) for the $P$. roqueforti enzyme are directly relevant to the A. terreus enzyme. Specifically, aromatic residues F112, F178, and W334 of the $P$. roqueforti enzyme appear to be appropriately oriented to stabilize sites of developing partial positive charge as well as carbocation intermediates in catalysis. These residues are conserved in the A. terreus enzyme as F87, F153, and W308. The results of site-directed mutagenesis experiments suggest that W334 of the $P$. roqueforti enzyme and W308 of the A. terreus enzyme stabilize the eudesmane cation (38). Electrostatic stabilization of highly reactive carbocation intermediates through cation- $\pi$ interactions $(39,40)$ is a catalytic strategy adopted by all terpenoid cyclases $(9)$. Additionally, just as the active site contour of the $P$. roqueforti enzyme is well-suited for the binding of the eudesmane cation (Figure 1) with a trans-decalin configuration, so too is the active site of the A. terreus enzyme due to their similar active site contours (Figures 6a and $6 b)$. The active site of 5-epi aristolochene synthase must accommodate the cis-decalin configuration of the eudesmane cation with a significantly different active site contour (Figure 6c).

The phenolic side chain of Y92 in P. roqueforti aristolochene synthase, conserved as Y67 in the A. terreus enzyme, was initially hypothesized to be the active site general acid/base responsible for the protonation of the germacrene A intermediate and the deprotonation of the eudesmane cation (Figure 1) (13). However, site-directed mutagenesis experiments with Y92F aristolochene synthase from $P$. roqueforti reveal the generation of appreciable quantities of aristolochene $(21,41,42)$. Therefore, neither Y 92 of the $P$. roqueforti enzyme nor Y67 of the A. terreus enzyme can be the catalytically obligatory general acid/base. Since the product aristolochene fits so precisely into the active site cavity in the closed conformation (Figure 7), it is unlikely that a solvent molecule could be trapped in the enclosed active site to perform a general acid/base function. Therefore, based on inspection of the structure of the $\mathrm{Mg}^{2+}{ }_{3}-\mathrm{PP}_{\mathrm{i}}$ complex, we propose that the $\mathrm{PP}_{\mathrm{i}}$ moiety is the best candidate for the general acid/base.

Specifically, consider the deprotonation of $\mathrm{C} 8-\mathrm{H}_{\beta}$ in the eudesmane cation (Figure 1). The $\mathrm{O} 3$ and $\mathrm{O} 6$ atoms of $\mathrm{PP}_{\mathrm{i}}$ are oriented into the active site cavity; based on comparison with trichodiene synthase (36) and bornyl diphosphate synthase (30), the O6 atom corresponds to the phosphoester oxygen and the $\mathrm{O} 3$ atom corresponds to the terminal phosphate group of the substrate farnesyl diphosphate. In the aristolochene synthase complex with $\mathrm{Mg}^{2+}{ }_{3}-\mathrm{PP}_{\mathrm{i}}$, the $\mathrm{O} 3$ and $\mathrm{O} 6$ atoms of $\mathrm{PP}_{\mathrm{i}}$ are $3.2 \AA$ and $4.6 \AA$ away, respectively, from the aristolochene $\mathrm{C} 8$ atom when the product is modeled into its complementary surface contour (Figure 7). If the bicyclic eudesmane cation binds in a position and orientation comparable to that modeled for the bicyclic product, the $\mathrm{O} 3$ atom of $\mathrm{PP}_{\mathrm{i}}$ would be properly oriented for the stereospecific deprotonation of $\mathrm{C} 8-\mathrm{H}_{\beta}$ in the eudesmane cation. 
In the preceding step, the conjugate acid of $\mathrm{PP}_{\mathrm{i}}$ could protonate the intermediate germacrene $\mathrm{A}$ at $\mathrm{C} 6$ to generate the eudesmane cation in the preceding step (Figure 1), since the $\mathrm{O} 3$ atom of $\mathrm{PP}_{\mathrm{i}}$ is also $3.4 \AA$ away from the corresponding atom of aristolochene when the product is modeled into its complementary surface contour (Figure 7). However, the origin of such a proton might be unclear. Experiments with [12, 12, $\left.12-{ }^{2} \mathrm{H}_{3}\right]$-farnesyl diphosphate (18) do not indicate any return of the original deuterium to $\mathrm{C} 6$ in the protonation of germacrene A, suggesting that the substrate deprotonation in the first step of the cyclization reaction does not involve the single general acid/general base invoked in Figure 1 unless there is competing rapid exchange of the $\mathrm{PP}_{\mathrm{i}}$-bound proton with the external medium, e.g., across metal-bound solvent molecules observed in the $\mathrm{Mg}^{2+}{ }_{3}-\mathrm{PP}_{\mathrm{i}}$ complex. It is conceivable that following ionization of farnesyl diphosphate, $\mathrm{O} 6$ of the newly generated $\mathrm{Mg}^{2+}{ }_{3}-\mathrm{PP}_{\mathrm{i}}$ deprotonates the paired germacradienyl cation, with the distal $\mathrm{O} 3$ of the $\mathrm{PP}_{\mathrm{i}}$ then donating a different proton to $\mathrm{C} 6$ of the intermediate germacrene A.

Although we previously speculated that the $\mathrm{PP}_{\mathrm{i}}$ anion might be involved in catalysis of the cyclization reaction, based on analysis of the structure of unliganded aristolochene synthase from $P$. roqueforti (13), the structure of A. terreus aristolochene synthase complexed with $\mathrm{Mg}^{2+}{ }_{3}-\mathrm{PP}_{\mathrm{i}}$ provides the first structural evidence that the $\mathrm{PP}_{\mathrm{i}}$ anion is indeed positioned and oriented to function as a possible general acid/general base for catalysis. This function is comparable to that recently proposed for the $\mathrm{PP}_{\mathrm{i}}$ anion in the termination step of the cationic chain elongation reaction catalyzed by farnesyl diphosphate synthase (43). Accordingly, aristolochene synthase may do relatively little in catalysis other than to fold the substrate farnesyl diphosphate into a suitable conformation and then trigger substrate ionization, while providing a solvent-shielded template for the ensuing cyclization cascade and allowing for substrate-assisted catalysis by the pyrophosphate leaving group. Consistent with such a catalytic strategy, it is notable that the slowest chemical step in sesquiterpene cyclization reactions is typically the ionization of the farnesyl diphosphate substrate $\left(1-5 \mathrm{~s}^{-1}\right)$, with all subsequent cyclizations, rearrangements, and deprotonations occuring as much as 100 times more rapidly $\left(>200 \mathrm{~s}^{-1}\right)(44,45)$. It is intriguing to speculate that such a catalytic strategy may be widespread among the greater family of terpenoid cyclases.

\section{Acknowledgements}

We thank the National Synchrotron Light Source at Brookhaven National Laboratory for access to X-ray crystallographic data collection facilities at beamline X29A and the Cornell High Energy Synchrotron Source for access to beamline F-1. We also thank L. Sangeetha Vedula for helpful advice.

\section{References}

1. Glasby, JS. Encyclopedia of Terpenoids. Wiley; Chichester: 1982.

2. Pichersky E, Noel JP, Dudareva N. Biosynthesis of plant volatiles: Nature's diversity and ingenuity. Science 2006;311:808-811. [PubMed: 16469917]

3. Cane DE. Isoprenoid biosynthesis. Stereochemistry of the cyclization of allylic pyrophosphates. Acc Chem Res 1985;18:220-226.

4. Cane DE. Enzymatic formation of sesquiterpenes. Chem Rev 1990;90:1089-1103.

5. Lesburg CA, Caruthers JM, Paschall CM, Christianson DW. Managing and manipulating carbocations in biology: Terpenoid cyclase structure and mechanism. Curr Opin Struct Biol 1998;8:695-703. [PubMed: 9914250]

6. Wendt KU, Schulz GE. Isoprenoid biosynthesis: Manifold chemistry catalyzed by similar enzymes. Structure 1998;6:127-133. [PubMed: 9519404]

7. Wise, ML.; Croteau, R. Monoterpene biosynthesis. In: Cane, DE., editor. Comprehensive Natural Products Chemistry: Isoprenoids Including Carotenoids and Steroids. 2. Elsevier; Oxford: 1999. p. 97-153. 
8. Cane, DE. Sesquiterpene biosynthesis: cyclization mechanisms. In: Cane, DE., editor. Comprehensive Natural Products Chemistry: Isoprenoids Including Carotenoids and Steroids. 2. Elsevier; Oxford: 1999. p. 155-200.

9. Christianson DW. Structural biology and chemistry of the terpenoid cyclases. Chem Rev 2006;106:3412-3442. [PubMed: 16895335]

10. Steele CL, Crock J, Bohlmann J, Croteau R. Sesquiterpene synthases from Grand fir (Abies grandis). Comparison of constitutive and wound-induced activities, and cDNA isolation, characterization, and bacterial expression of delta-selinene synthase and gamma-humulene synthase. J Biol Chem 1998;273:2078-2089. [PubMed: 9442047]

11. Starks CM, Back K, Chappell J, Noel JP. Structural basis for cyclic terpene biosynthesis by tobacco 5-epi-aristolochene synthase. Science 1997;277:1815-1820. [PubMed: 9295271]

12. Lesburg CA, Zhai G, Cane DE, Christianson DW. Crystal structure of pentalenene synthase: mechanistic insights on terpenoid cyclization reactions in biology. Science 1997;277:1820-1824. [PubMed: 9295272]

13. Caruthers JM, Kang I, Rynkiewicz MJ, Cane DE, Christianson DW. Crystal structure determination of aristolochene synthase from blue cheese mold, Penicillium roqueforti. J Biol Chem 2000;275:25533-25539. [PubMed: 10825154]

14. Rynkiewicz MJ, Cane DE, Christianson DW. Structure of trichodiene synthase from Fusarium sporotrichioides provides mechanistic inferences on the terpene cyclization cascade. Proc Natl Acad Sci USA 2001;98:13543-13548. [PubMed: 11698643]

15. Ashby MN, Edwards PA. Elucidation of the deficiency in two yeast coenzyme Q mutants. Characterization of the structural gene encoding hexaprenyl pyrophosphate Synthetase. J Biol Chem 1990;265:13157-13164. [PubMed: 2198286]

16. Cane DE, Kang I. Aristolochene synthase: purification, molecular cloning, high-level expression in Escherichia coli, and characterization of the Aspergillus terreus cyclase. Arch Biochem Biophys 2000;376:354-364. [PubMed: 10775423]

17. Cane DE, Prabhakaran PC, Salaski EJ, Harrison PHM, Noguchi H, Rawlings BJ. Aristolochene biosynthesis and enzymatic cyclization of farnesyl pyrophosphate. J Am Chem Soc 1989;111:89148916.

18. Cane DE, Prabhakaran PC, Oliver JS, McIlwaine DB. Aristolochene biosynthesis. Stereochemistry of the deprotonation steps in the enzymatic cyclization of farnesyl pyrophosphate. J Am Chem Soc 1990;112:3209-3210.

19. Cane DE, Tsantrizos YS. Aristolochene synthase. Elucidation of the cryptic germacrene A synthase activity using the anomalous substrate dihydrofarnesyl diphosphate. J Am Chem Soc 1996;118:10037-10040.

20. Cane DE, Bryant C. Aristolochene synthase. Mechanism-based inhibition of a terpenoid cyclase. J Am Chem Soc 1994;116:12063-12064.

21. Felicetti B, Cane DE. Aristolochene synthase: mechanistic analysis of active site residues by sitedirected mutagenesis. J Am Chem Soc 2004;126:7212-7221. [PubMed: 15186158]

22. Otwinowski Z, Minor W. Processing of X-ray diffraction data collected in oscillation mode. Meth Enzymol 1997;276:306-326.

23. McCoy AJ, Grosse-Kunstleve RW, Storoni LC, Read RJ. Likelyhood-enhanced fast translation functions. Acta Crystallogr sect D 2005;61:458-464. [PubMed: 15805601]

24. Brünger AT, Adams PD, Clore GM, DeLano WL, Gros P, Grosse-Kunstleve RW, Jiang JS, Kuszewski J, Nilges M, Pannu NS, Read RJ, Rice LM, Simonson T, Warren GL. Crystallography and NMR system: a new software suite for macromolecular structure determination. Acta Crystallogr sect D 1998;54:905-921. [PubMed: 9757107]

25. Jones TA, Zou JY, Cowan SW, Kjeldgaard M. Improved methods for building protein models in electron density maps and the location of errors in these models. Acta Crystallogr sect A 1991;47:110-119. [PubMed: 2025413]

26. Lee B, Richards FM. The interpretation of protein structures: estimation of static accessibility. J Mol Biol 1971;55:379-400. [PubMed: 5551392]

27. Kleywegt GJ, Jones TA. Detection, delineation, measurement and display of cavities in macromolecular structures. Acta Crystallogr sect D 1994;50:178-185. [PubMed: 15299456] 
28. Tarshis LC, Yan M, Poulter D, Sacchettini JC. Crystal structure of recombinant farnesyl diphosphate synthase at 2.6-Å resolution. Biochemistry 1994;33:10871-10877. [PubMed: 8086404]

29. Ponstingl H, Kabir T, Gorse D, Thornton JM. Morphological aspects of oligomeric protein structures. Prog Biophys Mol Biol 2005;89:9-35. [PubMed: 15895504]

30. Whittington DA, Wise ML, Urbansky M, Coates RM, Croteau RB, Christianson DW. Bornyl diphosphate synthase: structure and strategy for carbocation manipulation by a terpenoid cyclase. Proc Natl Acad Sci USA 2002;99:15375-15380. [PubMed: 12432096]

31. Cane DE, Xue Q, Fitzsimons BC. Trichodiene synthase. Probing the role of the highly conserved aspartate-rich region by site-directed mutagenesis. Biochemistry 1996;35:12369-12376. [PubMed: 8823172]

32. Rynkiewicz MJ, Cane DE, Christianson DW. X-ray crystal structures of D100E trichodiene synthase and its pyrophosphate complex reveal the basis for terpene product diversity. Biochemistry 2002;41:1732-1741. [PubMed: 11827517]

33. Cane DE, Shim JH, Xue Q, Fitzsimons BC. Trichodiene synthase. Identification of active site residues by site-directed mutagenesis. Biochemistry 1995;34:2480-2488. [PubMed: 7873527]

34. Cane DE, Xue Q. Trichodiene synthase. Enzymatic formation of multiple sesquiterpenes by alteration of the cyclase active site. J Am Chem Soc 1996;118:1563-1564.

35. Vedula LS, Rynkiewicz MJ, Pyun H-J, Coates RM, Cane DE, Christianson DW. Molecular recognition of the substrate diphosphate group governs product diversity in trichodiene synthase mutants. Biochemistry 2005;44:6153-6163. [PubMed: 15835903]

36. Vedula LS, Cane DE, Christianson DW. Role of arginine-304 in the diphosphate-triggered active site closure mechanism of trichodiene synthase. Biochemistry 2005;44:12719-12727. [PubMed: 16171386]

37. Yoshikuni Y, Ferrin TE, Keasling JD. Designed divergent evolution of enzyme function. Nature 2006;440:1078-1082. [PubMed: 16495946]

38. Deligeorgopoulou A, Taylor SE, Forcat S, Allemann RK. Stabilisation of eudesmane cation by tryptophan 334 during aristolochene synthase catalysis. Chem Commun (Camb) 2003;17:21622163. [PubMed: 13678181]

39. Dougherty DA. Cation-pi interactions in chemistry and biology: a new view of benzene, Phe, Tyr, and Trp. Science 1996;271:163-168. [PubMed: 8539615]

40. Jenson C, Jorgensen WL. Computational investigations of carbenium ion reactions relevant to sterol biosynthesis. J Am Chem Soc 1997;119:10846-10854.

41. Calvert MJ, Ashton PR, Allemann RK. Germacrene A is a product of the aristolochene synthasemediated conversion of farnesyl pyrophosphate to aristolochene. J Am Chem Soc 2002;124:1163611641. [PubMed: 12296728]

42. Deligeorgopoulou A, Allemann RK. Evidence for differential folding of farnesyl pyrophosphate in the active site of aristolochene synthase: a single-point mutation converts aristolochene synthase into an (E)- $\beta$-farnesene synthase. Biochemistry 2003;42:7741-7747. [PubMed: 12820883]

43. Hosfield DJ, Zhang Y, Dougan DR, Broun A, Tari LW, Swanson RV, Finn J. Structural basis for bisphosphonate-mediated inhibition of isoprenoid biosynthesis. J Biol Chem 2004;279:8526-8529. [PubMed: 14672944]

44. Cane DE, Chiu H-T, Liang P-H, Anderson KS. Pre-steady-state kinetic analysis of the trichodiene synthase reaction pathway. Biochemistry 1997;36:8332-8339. [PubMed: 9204880]

45. Mathis JR, Back K, Starks C, Noel J, Poulter CD, Chappell J. Pre-steady-state study of recombinant sesquiterpene cyclases. Biochemistry 1997;36:8340-8348. [PubMed: 9204881] 


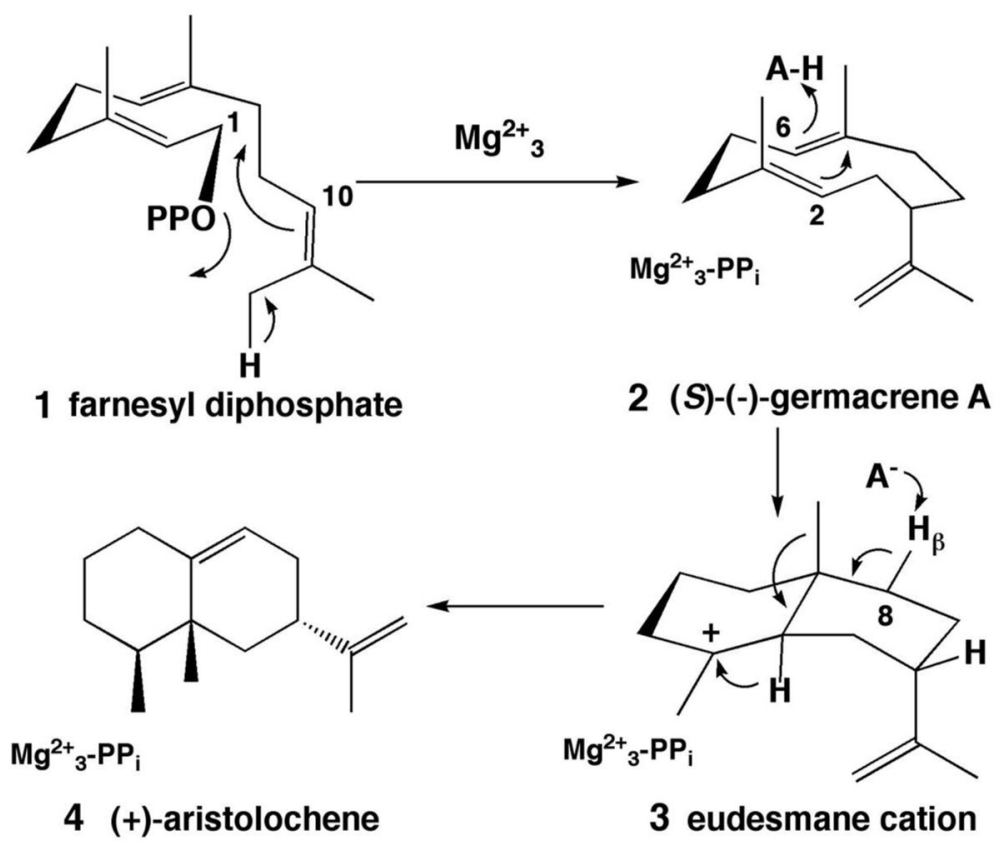

Figure 1.

Cyclization of farnesyl diphosphate (1) to form (+)-aristolochene (4). Mechanistic details are summarized in the text; the pyrophosphate leaving group of the substrate may serve as a general acid/general base in catalysis. 
a

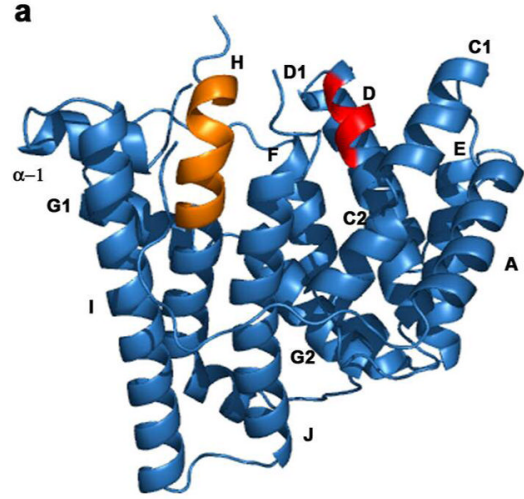

b
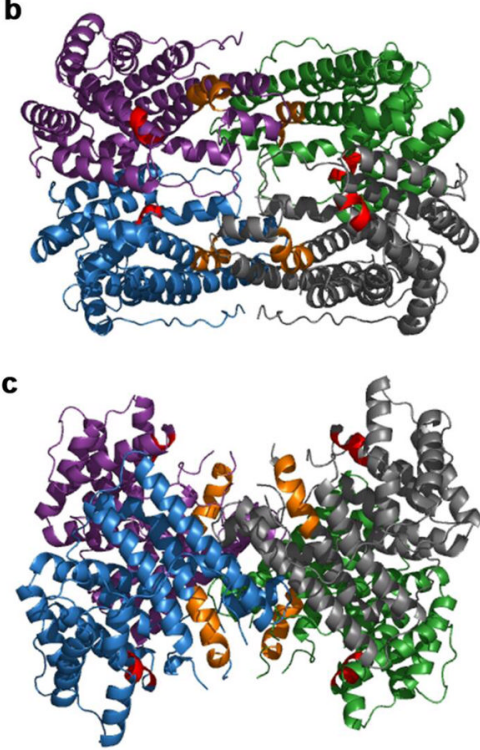
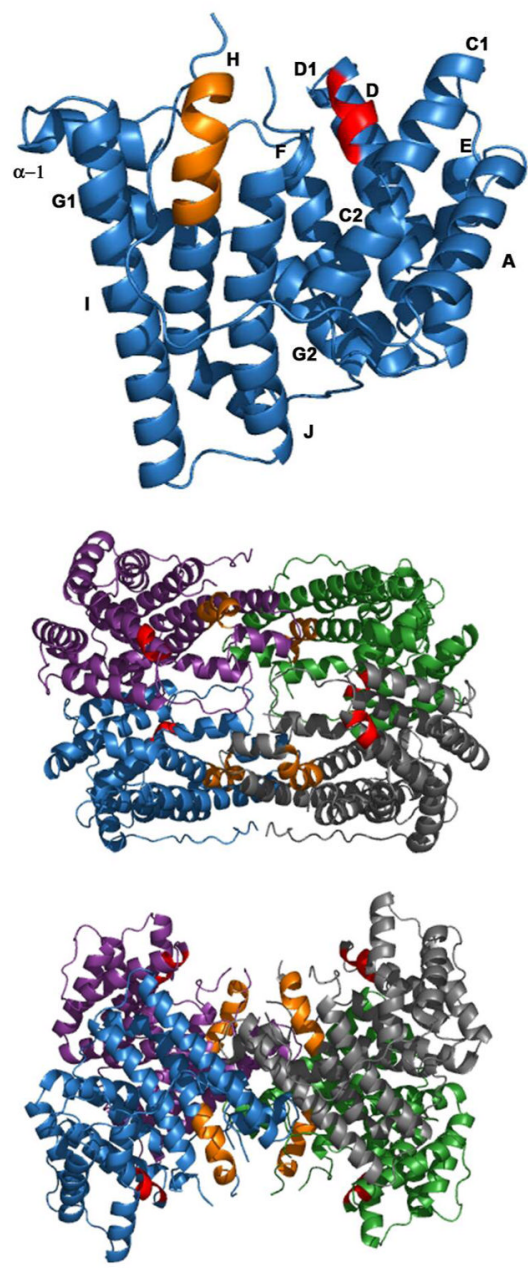

Figure 2.

Ribbon plots of A. terreus aristolochene synthase. (a) Stereoview of cyclase monomer A showing the aspartate-rich motif (red) and the aspartate-poor NSE motif (orange) flanking the mouth of the active site. Helices are labeled according to the convention first established for farnesyl diphosphate synthase (28). (b) Cyclase monomers A (blue), B (green), C (purple), and $\mathrm{D}$ (grey) assemble to form a dimer of dimers. (c) Same as (b) but rotated by $90^{\circ}$. 


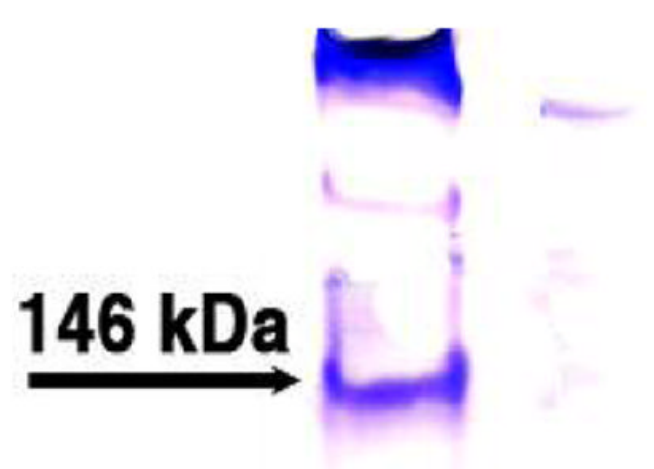

\section{$\stackrel{66 \mathrm{kDa}}{\longrightarrow}$}

\section{$20 \mathrm{kDa}$}

\section{$\begin{array}{llll}\text { Std } & 1 & 2 & 3\end{array}$}

Figure 3.

Native gel analysis of $A$. terreus aristolochene synthase oligomerization in solution. Novex Tris-glycine sample native buffer was added to $28 \mu \mathrm{M}, 20 \mathrm{nM}$, and $11 \mathrm{mM}$ enzyme in $25 \mathrm{mM}$ MES (pH 6.5), 2 mM MgCl $2,0.15 \mathrm{M} \mathrm{NaCl}, 4$ mM BME. The NativeMark Unstained protein standard from Invitrogen and enzyme samples were electrophoresed on 10-20\% native Tris Glycine gel at $125 \mathrm{~V}$ and $4{ }^{\circ} \mathrm{C}$ in $50 \mathrm{mM}$ Tris-glycine buffer ( $\mathrm{pH}$ 8.8) for approximately 5 hours. Lanes labeled 1,2 and 3 indicate $28 \mu \mathrm{M}, 20 \mathrm{nM}$, and $11 \mathrm{mM}$ aristolochene synthase, respectively. The native enzyme migrates as a $\sim 70 \mathrm{kDa}$ dimer. 


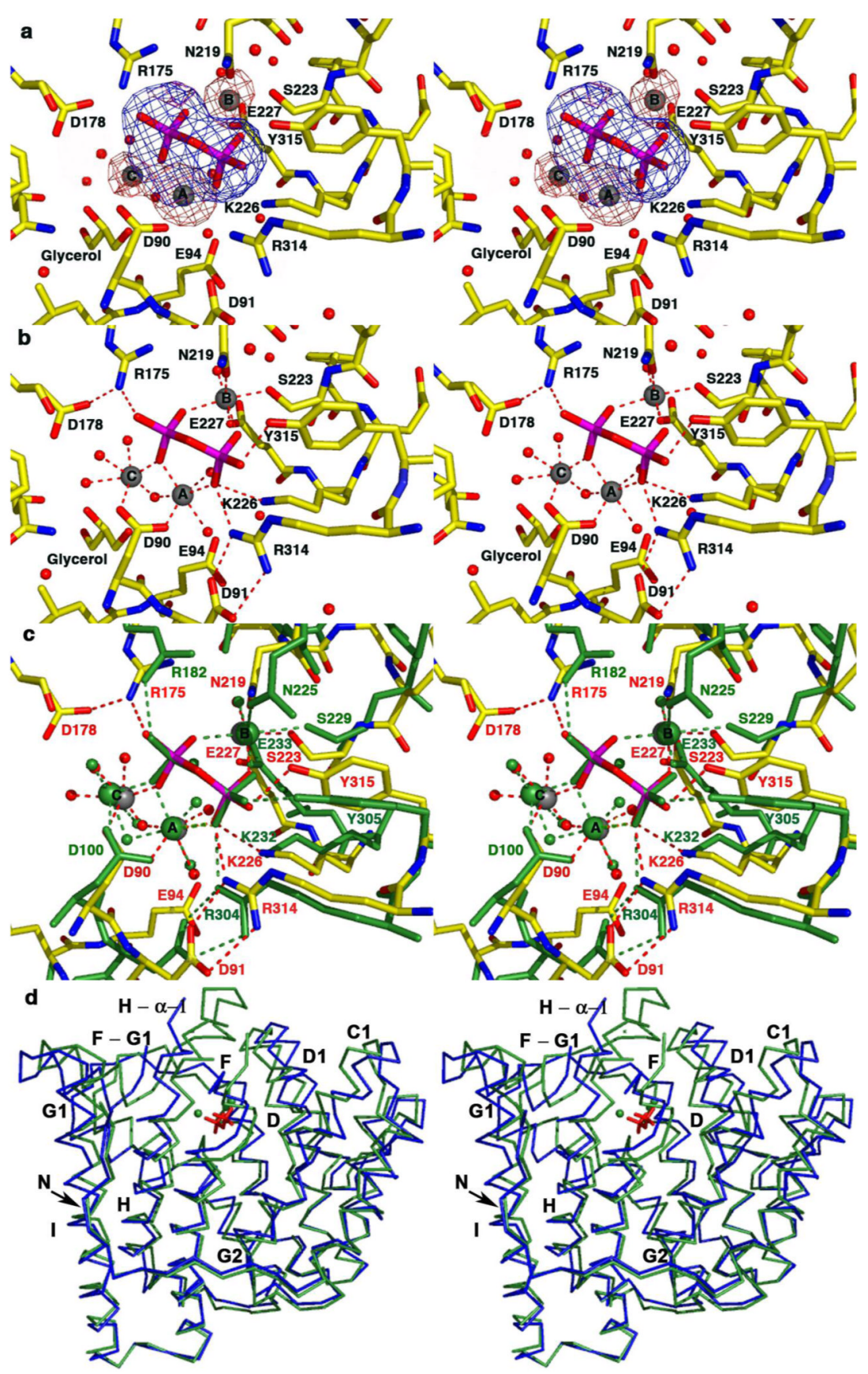

Figure 4.

Aristolochene synthase- $-\mathrm{Mg}^{2+}{ }_{3}-\mathrm{PP}_{\mathrm{i}}$ complex. (a) Simulated annealing omit maps of the $\mathrm{PP}_{\mathrm{i}}$ anion (blue) and $\mathrm{Mg}^{2+}$ ions (red) contoured at $3 \sigma$. The $\mathrm{Mg}^{2+}$ ions are shown as grey spheres and water molecules appear as red spheres. (b) Same orientation as (a) showing selected metal coordination and hydrogen bond interactions. (c) Superposition of aristolochene synthase and trichodiene synthase (green) active sites in their $\mathrm{Mg}^{2+}{ }_{3}-\mathrm{PP}_{\mathrm{i}}$ complexes. (d) Least-square superposition of $300 \mathrm{C} \alpha$ atoms between unliganded (blue) and $\mathrm{Mg}^{2+}{ }_{3}$-complexed (green) aristolochene synthase. Glycerol molecules are omitted from both structures for clarity. Significant conformational changes are triggered in the indicated helices and loops by $\mathrm{Mg}^{2+}{ }_{3}-\mathrm{PP}_{\mathrm{i}}$ binding. 
a
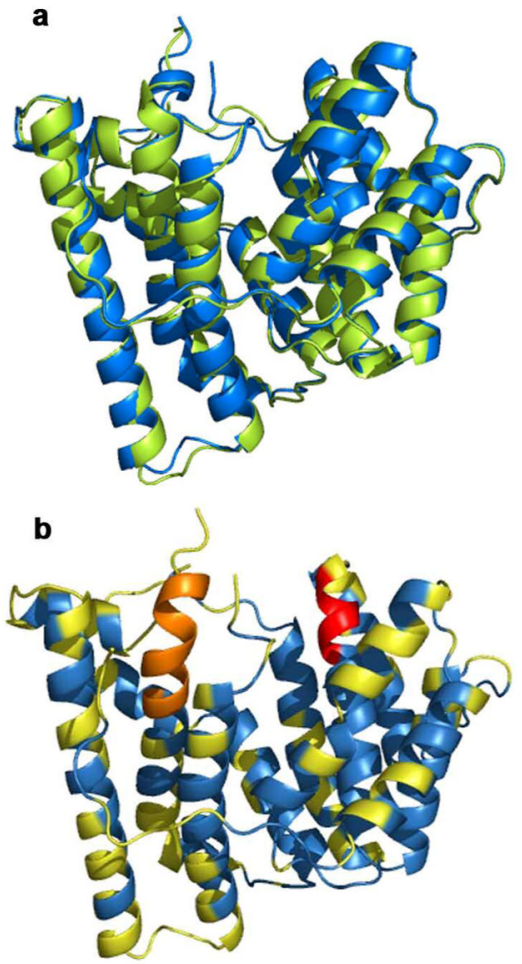
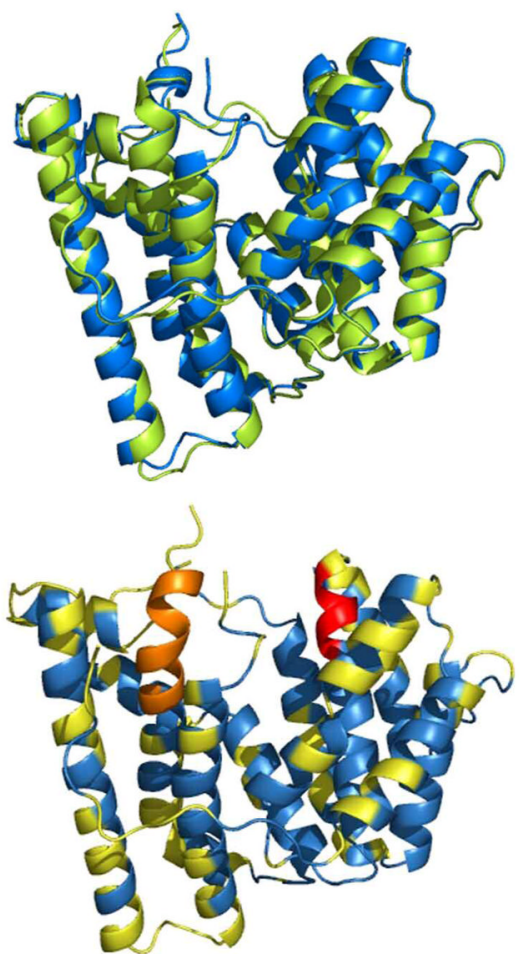

Figure 5.

(a) Superposition of aristolochene synthases from A. terreus (blue) and P. roqueforti (green). Aristolochene synthase from $P$. roqueforti crystallizes as a dimer along a two-fold noncrystallographic axis. With $1630 \AA^{2}$ buried surface area, the dimer interface is identical to those between the $\mathrm{AD}$ and $\mathrm{BC}$ monomer pairs in the A. terreus enzyme. (b) Stereoview of aristolochene synthase from $A$. terreus with sites of amino acid substitutions in the $P$. roqueforti enzyme colored yellow. Aspartate-rich and NSE motifs are red and orange, respectively. 

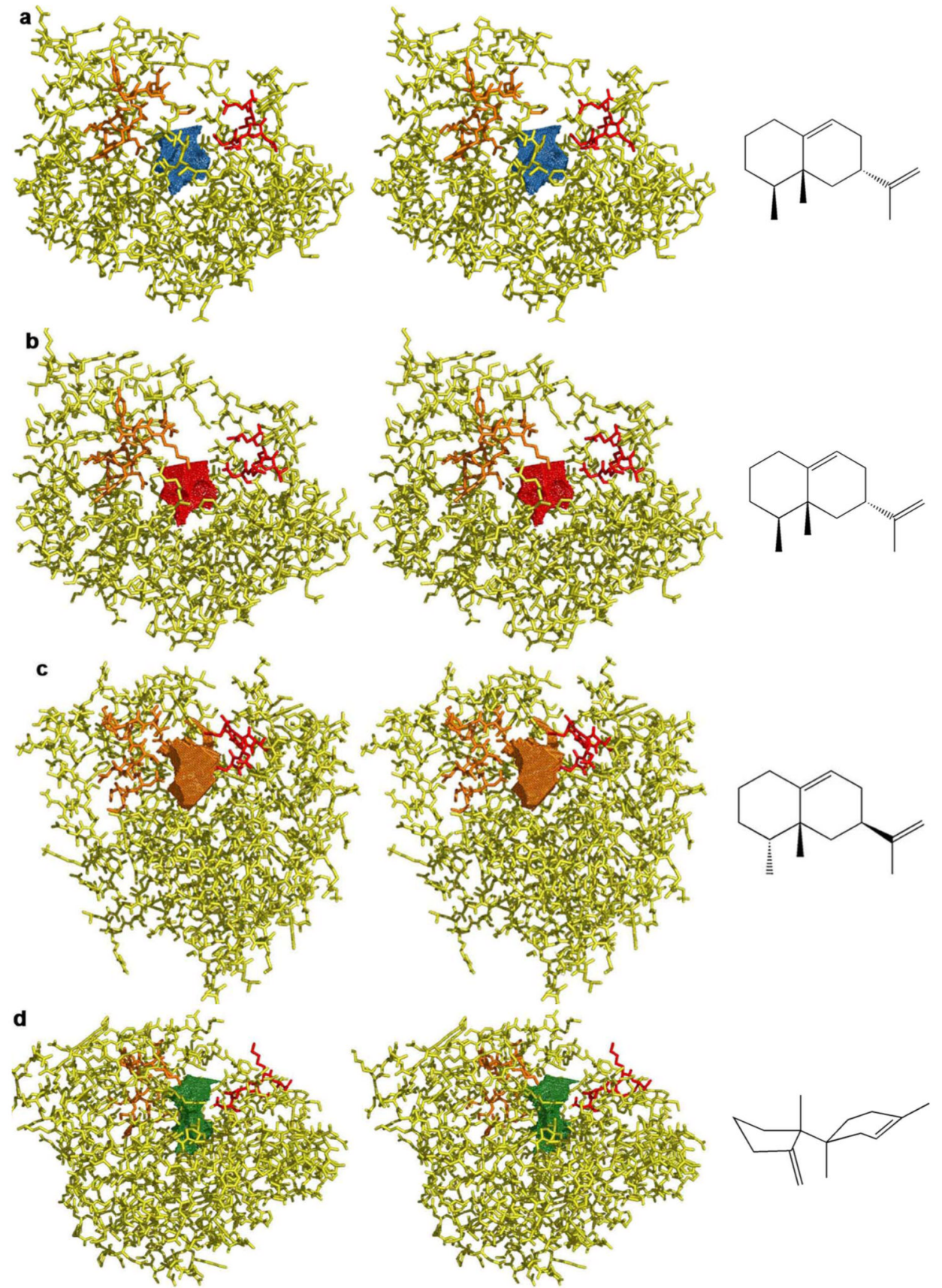

Figure 6.

Left: stereoviews of sesquiterpene cyclases and their solvent accessible active site surface contours in the open, unliganded conformations: (a) aristolochene synthase from A. terreus, (b) aristolochene synthase from $P$. roqueforti, (c) C-terminal domain of 5-epi aristolochene synthase, (d) trichodiene synthase. Aspartate-rich motifs (red) and NSE motifs (orange) indicate the comparable orientations of active site clefts, roughly comparable to that shown in Figure 2a for aristolochene synthase from A. terreus. Right: cyclic sesquiterpenes generated by each enzyme. 

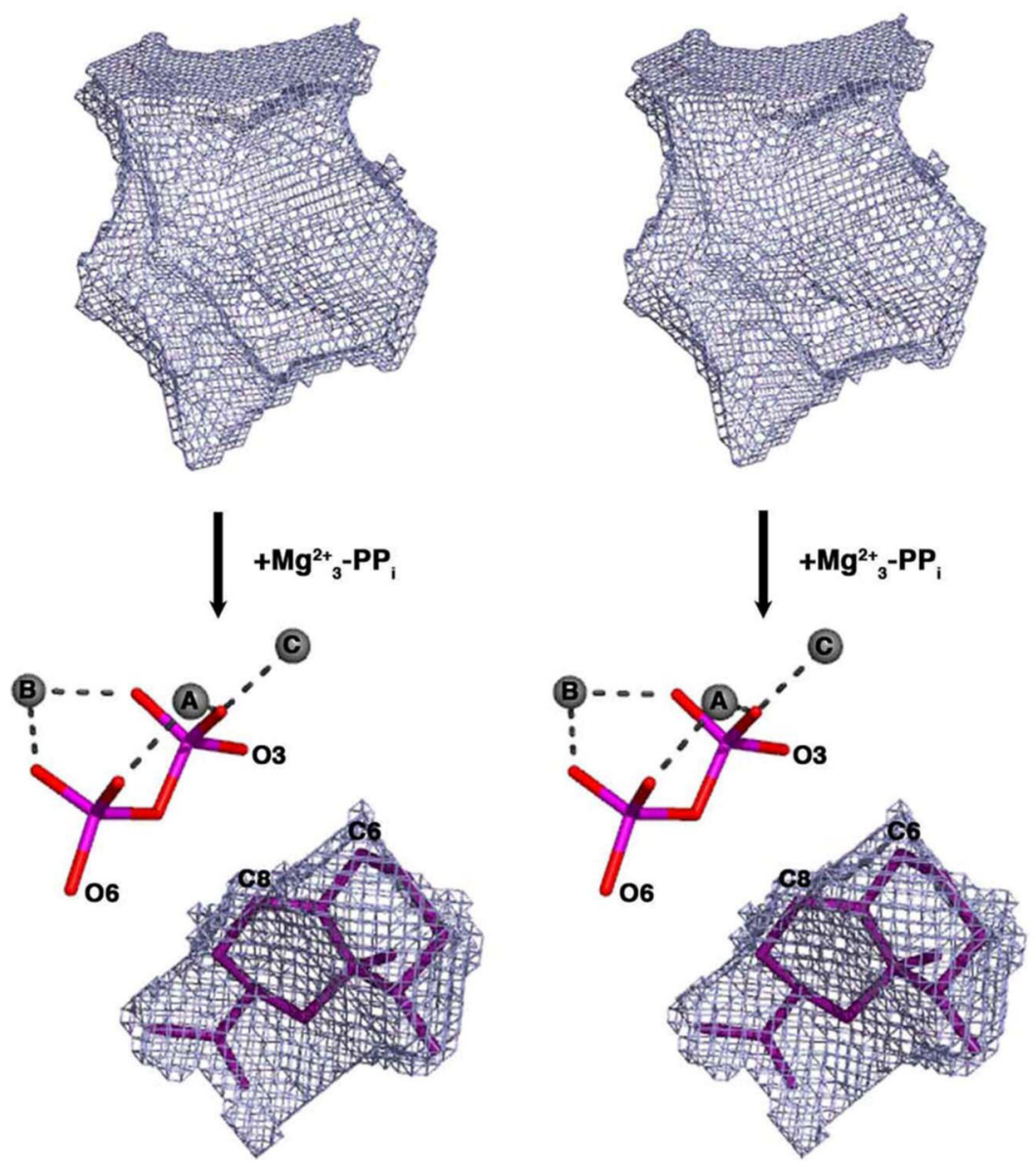

Figure 7.

Active site surface contours of $A$. terreus aristolochene synthase in the unliganded (from Figure $6 a)$ and liganded (closed) conformation. The cyclization product, (+)-aristolochene, is modeled into the active site of the closed conformation, and the location of the $\mathrm{Mg}^{2+}{ }_{3}-\mathrm{PP}_{\mathrm{i}}$ cluster is shown as a visual reference. The unique template required for the cyclization of farnesyl diphosphate is preserved in the lower active site contour in both open and closed conformations. The $\mathrm{O} 6$ atom of $\mathrm{PP}_{\mathrm{i}}$ corresponds to the original phosphoester oxygen and the $\mathrm{O} 3$ atom corresponds to the terminal phosphate group of the substrate, farnesyl diphosphate. The proximity of the $\mathrm{PP}_{\mathrm{i}} \mathrm{O} 3$ atom to aristolochene $\mathrm{C} 6$ and $\mathrm{C} 8$ atoms (3.4 $\AA$ and $3.2 \AA$, respectively) implicates $\mathrm{PP}_{\mathrm{i}}$ as the general acid/general base illustrated in Figure 1. 
Table 1

Data Collection and Refinement Statistics

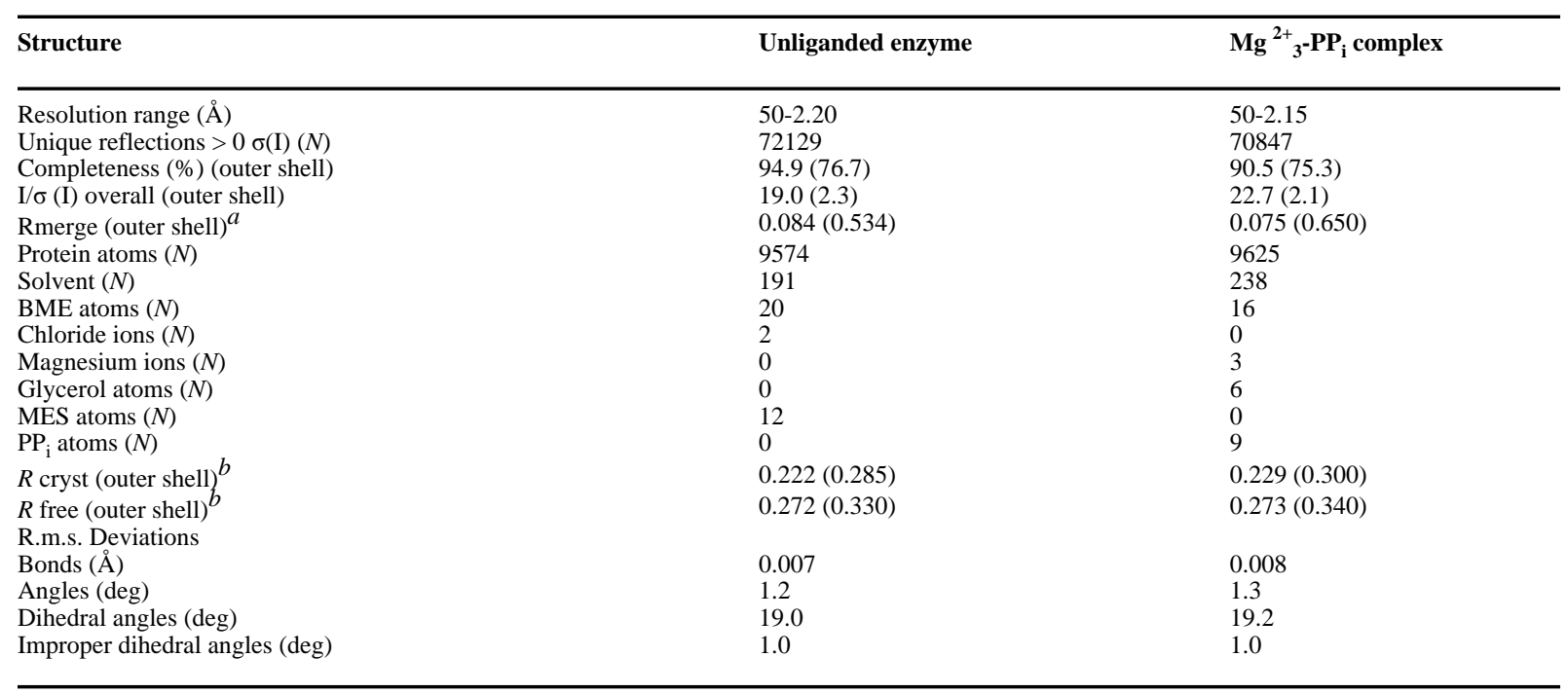

${ }^{a} R_{\text {merge }}=\Sigma\left|I_{i}-\left\langle I_{i}\right\rangle\right| / \Sigma I_{i}$, where $I_{i}$ is the observed intensity for reflection $i$ and $\left\langle I_{i}\right\rangle$ is the average intensity calculated for reflection $i$ from replicate data.

${ }^{b} R=\Sigma|| F_{\mathrm{O}}|-| F_{\mathrm{C}} \| / \Sigma\left|F_{\mathrm{O}}\right|$, where $R$ and $R$ free are calculated using the working and test reflection sets, respectively. 


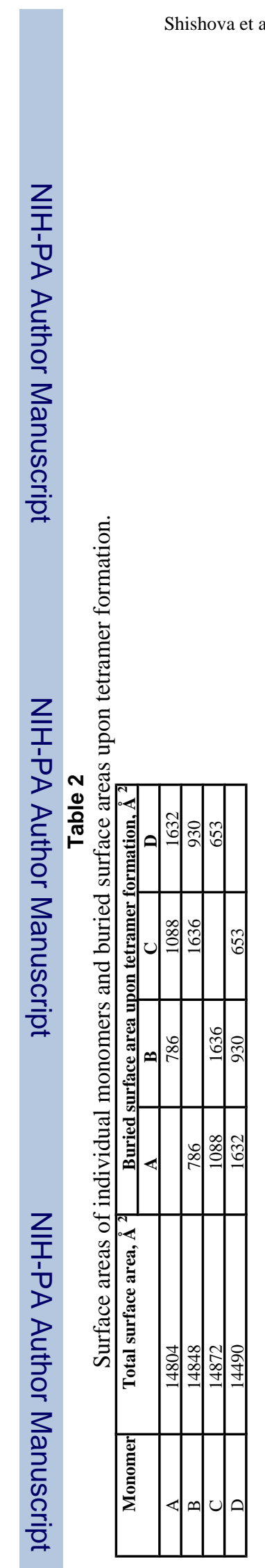

Biochemistry. Author manuscript; available in PMC 2008 August 21. 